\title{
Intracranial abscess due to Mycobacterium avium complex in an immunocompetent host: a case report
}

\author{
Mudit Chowdhary ${ }^{1 *}$, Umesh Narsinghani ${ }^{2}$ and Ritu A. Kumar ${ }^{1,3}$
}

\begin{abstract}
Background: Mycobacterium avium complex (MAC) is a ubiquitous pathogen, widely distributed in the environment including water, soil and animals. It is an uncommonly encountered clinical pathogen; primarily causing pulmonary infections in patients with underlying lung disease or disseminated disease in immunocompromised hosts. Sporadically, extra-pulmonary infections have been documented including involvement of the liver, spleen, skin, soft tissue and lymph nodes. Central nervous system (CNS) infections due to MAC are exceedingly rare and carry a poor prognosis. Additionally, such infections are largely reported in patients infected with HIV. Herein we report the first case of intracranial abscess due to MAC in an immunocompetent man with a normal CD4 count and negative HIV status.

Case presentation: A previously healthy 40-year-old male presented to us with progressively worsening CNS symptoms. The patient's presentation was uncharacteristic of MAC infection in immunocompetent hosts, as he developed subacute, progressive symptoms that included severe frontal headaches, left eyelid swelling, blurry vision, and diplopia, without any pulmonary or systemic manifestations. Neuroimaging revealed multiple ring-enhancing lesions, which required neurosurgical intervention. MAC was the only pathogen that grew from intraoperative tissue cultures. The patient was subsequently treated with a 12-month regimen consisting of Clarithromycin, Ethambutol, and Rifampin, with successful clinical resolution.
\end{abstract}

Conclusion: Our findings indicate that it is important to consider rare infections such as MAC in immunocompetent patients, regardless of atypical symptoms. Despite the severity of this infection, with timely diagnosis effective treatment is available.

Keywords: Mycobacterium avium, MAC, CNS, Brain abscess, Immunocompetent

\section{Background}

The most prevalent nontuberculous mycobacterium (NTM) capable of causing disease in humans is Mycobacterium avium complex (MAC) [1]. Organisms classified as MAC comprise of at least two related species namely Mycobacterium avium and Mycobacterium avium-intracellulare. These organisms have been recovered from water, soil, food and milk, domestic and wild animals, though they are readily cleared in most humans.

Infection with MAC can be acquired by ubiquitous environmental exposure. It most commonly causes pulmonary

\footnotetext{
*Correspondence: mu.chowdhary@gmail.com

'Department of Internal Medicine, Mercer University School of Medicine, 707 Pine Street, Macon, GA 31201, USA

Full list of author information is available at the end of the article
}

infections in patients with chronic lung diseases, and disseminated disease in the immunocompromised host $[1,2]$.

NTM infection, while an extremely rare cause for CNS infection, portends a significant mortality rate, ranging from $35-70 \%$ [3]. Studies have shown that disseminated disease, previous neurosurgery, and trauma are leading factors for CNS infection [2]. The majority of cases involving MAC infection of the CNS are seen as opportunistic infections in patients with acquired immunodeficiency syndrome (AIDS) with a severely depressed CD4 count $(<50$ cells/ $\mu \mathrm{l})[4]$.

Here, we report the first case of brain abscess caused by MAC in an HIV-negative male without any active underlying immunodeficiency.

\section{() Biomed Central}




\section{Case presentation}

A 40-year-old Caucasian male presented with a 6-week history of chronic unremitting frontal headaches, followed by left eyelid swelling, blurry vision, and diplopia. He subsequently developed nausea and vomiting. He denied any fever, nasal congestion, night sweats, or weight loss. His past medical history was significant for bipolar disorder with no other relevant findings. The patient was not using any current medications and he denied a history of immunosuppresive drugs. Moreover, his family history was only positive for hypertension, without any mention of autoimmune disease. On examination, the patient was afebrile, pulse was 69 per minute, respirations were 23 per minute, and blood pressure was 150/89 $\mathrm{mmHg}$.

The physical exam was notable for exophthalmos of the left eye as well as a palpable mass on the left eyelid. Neurological exam revealed third, fourth, and sixth cranial nerve palsy in the left eye. The patient also displayed decreased visual acuity in the affected eye with associated early disc edema. The remainder of the physical exam was unremarkable. The patient's complete and differential blood counts, serum chemistry and erythrocyte sedimentation rate were normal. Given the findings, the patient was referred for computed tomography (CT) to rule out a mass lesion.
CT of the orbit with contrast revealed two frontal lobe lesions, the largest of which appeared solid measuring $3.6 \times 3.8 \mathrm{~cm}$ on coronal image. The lesion was seen eroding through the orbital roof, exerting significant mass effect on the superior rectus muscle, and causing deformity of the left globe. An additional ring-enhancing left frontal lesion was seen adjacent to the solid tumor measuring $2.7 \times 1.9 \times 2.7 \mathrm{~cm}$.

Subsequent magnetic resonance imaging (MRI) of the brain with and without contrast revealed a mass arising in the left orbital roof with destruction of the sphenoid bone. Posterior to the mass was a round hyperintense cystic area within the left inferior frontal cortex with surrounding severe vasogenic edema. Mass effect was visualized with midline shift from left to right of the septum pellucidum and subfalcine herniation (Fig. 1). The contrast enhanced study showed an extensive lytic mass measuring at least $3.4 \times 2.2 \mathrm{~cm}$ in size. At the time, the etiology of the lesions was felt to be more likely neoplastic rather than infectious in the absence of any known immunocompromised state. Therefore, blood cultures were not obtained.

The patient consequently underwent a left frontal craniotomy for partial resection of the masses. Upon incision the large left frontal lobe lesion was full of purulent

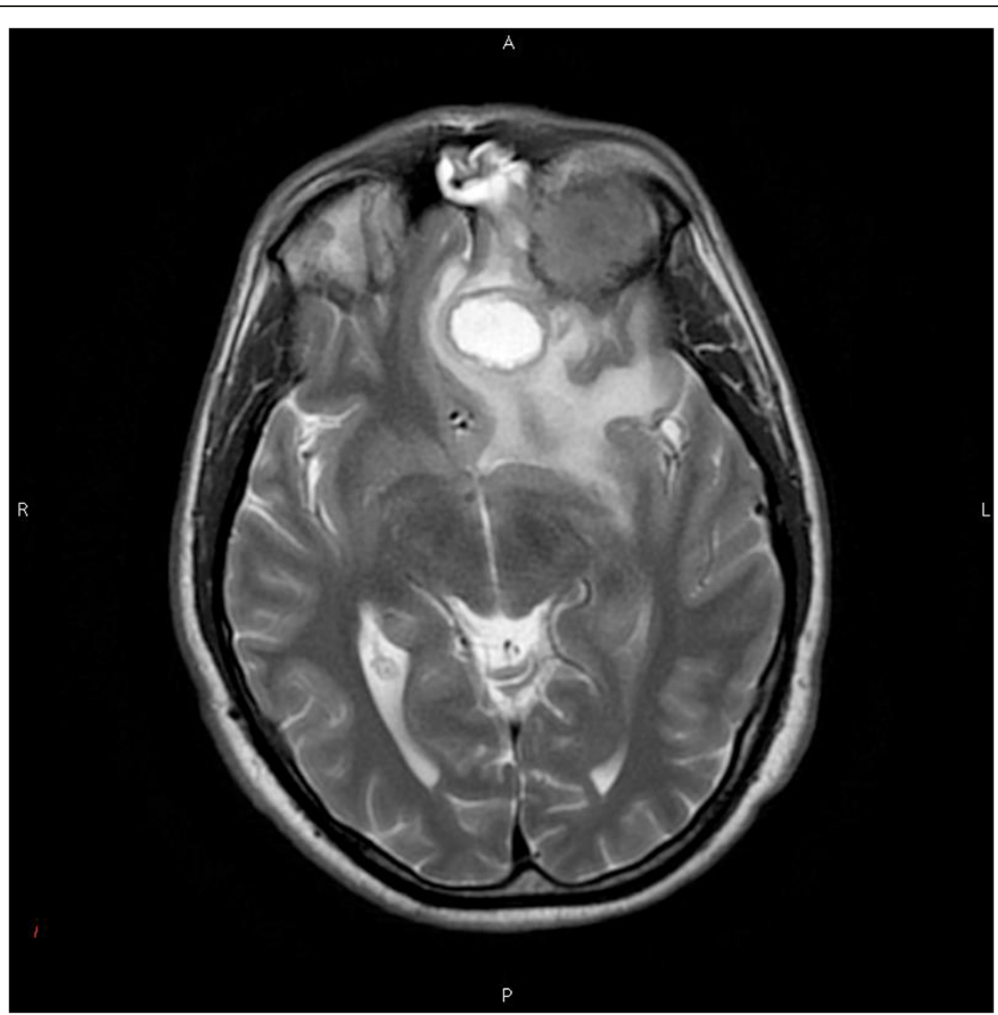

Fig. 1 Axial T2 MRI showing a round hyperintense cystic area with surrounding vasogenic edema, and orbital involvement along with left-to-right midline shift due to mass effect 
material from which multiple cultures were acquired. At this point, the differential favored intracranial abscess rather than neoplasm. Post-operative MRI noted that the previous ring-enhancing lesions were decreased in size. Additionally, the left retro-orbital hyperdense mass had resolved. The prior midline displacement was improved. Persistent vasogenic edema throughout the left frontal lobe was noted; however, the mass effect had resolved. Preliminary pathology from abscess resection revealed granulomatous inflammation and necrosis with positive acid-fast bacilli (AFB) cultures. Intraoperative bacterial, anaerobic and fungal cultures however were all negative. Routine blood cultures, AFB blood cultures and QuantiFERON $^{\circ}$ TB Gold test were also negative. The patient's sputum culture however was positive for AFB. Additionally, the patient was tested for HIV following abscess resection with negative results. Moreover, the patient's CD4 count was 515 cells $/ \mathrm{mm}^{3}$ (32\%). The patient was started on an anti-TB regimen consisting of Rifampin, Isoniazid, Pyrazinamide, and Ethambutol.

CT of the thorax was performed to rule out a concomitant pulmonary infection. The study demonstrated calcified bilateral hilar lymph nodes, as well as multiple calcified mediastinal lymph nodes involving the prevascular, pretracheal, precarinal, right paratracheal, and subcarinal space, reflecting prior granulomatous disease or sarcoidosis. Results from endobronchial biopsy revealed noncaseating granulomas. Final tissue cultures from the brain lesions and sputum revealed MAC. The patient was started on a 12-month MAC treatment regimen consisting of Clarithromycin, Ethambutol, and Rifampin. The patient's neurological symptoms, exopthalmous, diplopia and mass effect fully resolved over the following 6months. The blurry vision persisted, but was corrected with glasses. Sputum AFB was negative at 7 -months. Furthermore, follow-up MRI at 8-months was negative for intracranial abscess.

\section{Discussion}

Mycobacterium avium complex typically affects the lungs, but other manifestations have been described including involvement of spleen, mesenteric lymph nodes, liver, and intestines $[5,6,7]$. MAC infections have been diagnosed in patients without predisposing conditions or immunosuppression, but they typically present as focal pulmonary or gastrointestinal diseases or occasionally in disseminated forms usually sparing the CNS $[1,8,9]$. Disseminated disease may complicate MAC pulmonary disease through local multiplication and entry into the bloodstream with seeding of other organs.

The clinical presentation of MAC lung disease is nonspecific, highly variable and is influenced by whether the patient has pre-existing pulmonary disease. The most common clinical manifestations of disseminated MAC include intermittent or persistent fever, night sweats, weight loss, with additional symptoms of fatigue, malaise and anorexia. There has been no consistent immune deficiency identified to explain MAC infection in HIVnegative patients. However, apparent genetic defects of disseminated NTM infection have been associated with specific mutations in interferon (IFN) $\gamma$ and interleukin (IL)-12 synthesis and response pathways [10]. Additionally, specific non-immunocompromised groups have shown a predisposition for MAC pulmonary infection; reports show an increasing prevalence of bronchiectatic pulmonary MAC disease in elderly women without underlying risk factors [11].

Nevertheless, CNS infection due to MAC remains rare in patients with immunodeficiency, with even fewer case reports published on CNS infections due to MAC in immunocompetent patients. In 2011, a large-scale study was conducted to identify cases of CNS infections due to NTM. The authors identified only 15 out of 5960 $(<1 \%)$ patients with NTM CNS infection; 11 out of these 15 patients were HIV-seronegative. Among the patients, 1 had an infection due to Mycobacterium kansasii, 7 had infections due to Mycobacterium abscessus, and 1 presented with a mixed M. abscessus-Mycobacterium fortuitum infection. Only 2 cases were due to MAC (13\%) [3]. Furthermore, an extensive search in the English literature found only 8 additional cases with MAC brain abscess (Table 1): 7/8 patients were HIV-positive or presented with an underlying immunodeficiency $[4,9,12-17]$. Males were infected $75 \%$ of the time and the median age was 38 years. Additionally, the median CD4 count was only $90 \mathrm{~mm}^{3}$ (range $2-215 \mathrm{~mm}^{3}$ ). The additional immunocompetent case diagnosis is unclear as the patient presented with risk factors for MAC infection. That case described a patient with chronic granulomatous meningitis who subsequently developed a secondary MAC brain abscess as a consequence of immunosuppresion due to prednisone therapy [12]. Moreover, the CD4 count was unknown. Our patient however, presented with a normal CD4 count, negative HIV status and without any existing co-morbidities.

Diagnosis of CNS MAC may be difficult and challenging given the rarity of the condition, the potential differential diagnosis of neoplasm as well as the varying presentations of MAC in the central nervous system $[9,12]$. Patients presenting with focal neurologic symptoms and signs of headache, visual impairment, ophthalmoplegia, or papilledema warrant urgent evaluation. MRI is superior to $\mathrm{CT}$ in distinguishing suspected brain abscess, but culture of the specimen is the gold standard for accurate diagnosis $[9,18]$. The specimen should be sent for Gram's stain, aerobic, anaerobic, mycobacterial, and fungal cultures including histopathology. 
Table 1 Summary of previous cases in the English literature

\begin{tabular}{|c|c|c|c|c|c|c|}
\hline Case (Reference) & Age/Sex & $\mathrm{CD} 4 \mathrm{~mm}^{3}$ & $\mathrm{HIV}+/-$ & Site & Imaging & Comorbidity \\
\hline $1[4]$ & $42 / F$ & 14 & + & Fronto-parietal & $\begin{array}{l}\text { Single, ring enhancing mass, } \\
\text { with mass effect }\end{array}$ & Leptomeningitis \\
\hline $2[9]$ & $52 / \mathrm{M}$ & 175 & - & Occipital cerebellum & Single, ring enhancing mass & Tuberculosis sarcoidosis \\
\hline $3[11]$ & $38 / \mathrm{M}$ & 90 & - & Frontal, parietal & Multiple masses, edema & Sarcoidosis \\
\hline $4[12]$ & $31 / F$ & N/A & - & Temporal & Single mass, edema & MAC meningitis, prednisone therapy \\
\hline $5[13]$ & $35 / \mathrm{M}$ & 215 & + & Fronto-parietal & Single mass, edema & HAART, cryptococcal meningitis \\
\hline $6[14]$ & $40 / \mathrm{M}$ & 31 & + & Occipital & Single mass, edema & HAART, CMV, retinitis \\
\hline 7 [15] & $33 / \mathrm{M}$ & 2 & + & Frontal, parietal, occipital & Multiple masses, ring enhancing & Disseminated MAC \\
\hline $8[16]$ & $36 / \mathrm{M}$ & 170 & + & Temporal, temporo-parietal & Multiple masses & $\begin{array}{l}\text { HAART, Disseminated MAC, PCP, } \\
\text { oropharyngeal candidiasis }\end{array}$ \\
\hline Our case & $40 / \mathrm{M}$ & 515 & - & Frontal & $\begin{array}{l}\text { Single, ring enhancing mass, } \\
\text { edema and mass effect, } \\
\text { with invasion }\end{array}$ & - \\
\hline
\end{tabular}

MAC Mycobacterium avium complex, HAART Highly active antiretroviral therapy, CMV Cytolomegalovirus, PCP Pneumocystis pneumonia

Studies have failed to identify an optimal treatment regimen in MAC CNS infections, further complicating these cases. There is no relation between in vitro drug susceptibility and clinical response for agents other than the macrolides [19]. Clarithromycin is currently the only drug for which susceptibility testing is recommended, and is considered to be the mainstay of treatment in combination with other agents such as Ethambutol [19]. Other chemotherapeutic options for treatment of MAC include rifampin, rifabutin, aminoglycosides such as amikacin, and a flouroquinolone like moxifloxacin. The penetration of anti-NTM drugs to CNS infections is further complicated by the relatively impermeable bloodbrain barrier (BBB). Ethambutol and the macrolides only reach sufficient CSF concentrations in the presence of meningeal inflammation [20]. Although rifampin shows greater efficacy in penetrating the $\mathrm{BBB}$ than the other drugs, it may be below the minimum inhibitory concentration level for some microbial strains [20,21].

Guidelines from the Centers for Disease Control and Prevention, American Thoracic Society (ATS) and Infectious Diseases Society of America (IDSA) suggest initiating treatment with at least two drugs for disseminated MAC infection to prevent or delay the emergence of resistance [19]. Clarithromycin is the preferred first line agent with Ethambutol as the second recommended drug. Some clinicians may consider adding a third drug, either rifabutin or rifampin, in patients with disseminated MAC and HIV. Alternatively, azithromycin can be substituted for clarithromycin as it is efficacious and unlike clarithromycin, does not interact with rifabutin or rifampin in patients with AIDS [19]. In HIV patients, the United States Public Health Service and ATS/IDSA guidelines recommend minimum therapy for 12 months and 6 months of immune reconstitution [19]. With
MAC pulmonary disease, treatment should be continued until sputum cultures are consecutively negative for at least one year.

\section{Conclusion}

In summary, it is important to consider rare infections such as MAC in immunocompetent patients, regardless of atypical symptoms. We encourage clinicians to keep a high index of suspicion for this condition in order to prevent morbidity and mortality from disease progression. Furthermore, we stress the importance of obtaining tissue cultures in order to make a conclusive diagnosis. Combination drug therapy is essential to decrease the risk of resistance. Additionally, treatment with more than one agent is also associated with more rapid clearance of MAC from the bloodstream. Despite the severity of this infection, with timely diagnosis effective treatment is available.

\section{Consent}

Written informed consent was obtained from the patient for publication of this Case report and any accompanying images. A copy of the written consent is available for review by the Editor of this journal.

\section{Competing interests}

The authors declare that they have no competing interests.

\section{Authors' contributions}

RK conceived the idea of the case report and coordinated drafting the manuscript. MC, UN and RK participated in the literature review. All authors have contributed to the draft of the article, have read and approved the final manuscript.

\section{Acknowledgements}

The authors declare that funding was not utilized for the preparation of this manuscript. 


\section{Author details}

1Department of Internal Medicine, Mercer University School of Medicine, 707 Pine Street, Macon, GA 31201, USA. ²Department of Pediatrics, Mercer University School of Medicine, Macon, GA, USA. ${ }^{3}$ Department of Infectious Diseases, Mercer University School of Medicine, Macon, GA, USA.

Received: 5 March 2015 Accepted: 14 July 2015

Published online: 23 July 2015

\section{References}

1. Martins AB, Matos ED, Lemos ACM. Infection With the Mycobacterium avium Complex in Patients Without Predisposing Conditions - A Case Report and Literature Review. Braz J Infect Dis. 2005;9(2):173-9.

2. Talati NJ, Rouphael N, Kuppalli K, Franco-Paredes C. Spectrum of CNS disease caused by rapidly growing mycobacteria. Lancet Infect Dis. 2008;8:390-98

3. Lee $M$, Cheng $A$, Lee $Y$, Yang $C$, Lai $C$, Huang $Y$, et al. CNS infections caused by Mycobacterium abscessus complex: clinical features and antimicrobial susceptibilities of isolates. J Antimicrob Chemother. 2012;67:222-5.

4. Karne SS, Sangle SA, Kiyawat DS, Dharmashale SN, Kadam DB, Bhardwaj RS Mycobacterium avium-intracellularle brain absecess in HIV-positive patient. Ann Indian Acad Neurol. 2012;15:54-5.

5. Negatu Y, Mekonen E. A Case Report of Mycobacterium Avium Complex Peritonitis in an AIDS patient. Case Reports in Infectious Diseases. 2013;2013:590478.

6. Torriani FJ, McCutchan JA, Bozzette SA, Grafe MR, Havlir DY. Autopsy findings in AIDS patients with Mycobacterium avium complex bacteremia. J Infect Dis. 1994;170(6):1601-5.

7. Jr HRJ. Mycobacterium avium Complex Infection in the Acquired Immunodeficiency Syndrome. N Engl J Med. 1991;324:1332-8.

8. Kim W, Jang S, Ok T, Kim GU PH, Leem J, Kang BH, et al. Disseminated Mycobacterium intracellulare Infection in an Immunocompetent Host. Tuberc Respir Dis. 2012;72:452-6.

9. Arkun K, Gordon DA, Lincoln C, Levi M, Bello J, Keller CE, et al. Atypical mycobacterial brain abscess presenting as a spindle cell lesion in an immunocompetent patient. Clin Neuropathol. 2012;31(3):155-8.

10. O'Connell E, Rosen LB, Larue RW, Fabre V, Melia MT, Auwaerter PG, et al. Domestic Report of Disseminated Mycobacterium avium Complex and Anti-Interferon-y Autoantibodies. J Clin Immunol. 2014;34:928-32.

11. Lee G, Lee KS, Moon JW, Koh WJ, Jeong BH, Jeong YJ, et al. Nodular bronchiectatic Mycobacterium avium complex pulmonary disease. Natural course on serial computed tomographic scans. Ann Am Thoracic Soc. 2013;10(4):299-306.

12. Morrison A, Gyure KA, Stone J, Wong K, McEvoy P, Koeller K, et al. Mycobacterial spindle cell pseudotumor of the brain; a case report and review of the literature. Am J Surg Pathol. 1999;23:1294-9.

13. Uldry PA, Bogousslavsky J, Regli F, Chave JP, Beer V. Chronic Mycobacterium avium complex infection of the central nervous system in non-immunosuppressed woman. Eur Neurol. 1992;32:285-8.

14. Murray R, Mallal S, Heath C, French M. Cerebral mycobacterium avium infection in an HIV-infected patient following immune reconstitution and cessation of therapy for disseminated mycobacterium avium complex infection. Eur J Clin Microbiol Infect Dis. 2011;20:199-201.

15. Berger $P$, Lepidi H, Drogoul-Vey MP, Poizot-Martin I, Drancourt M. Mycobacterium avium brain abscess at the initiation of highly active antiretroviral therapy. Eur J Clin Microbiol Infect Dis. 2004;23:142-4.

16. Verma R, Dhamija R. Disseminated Mycobacterium avium-intracellulare infection presenting as multiple ring-enhancing lesions on brain MRI. Mayo Clin Proc. 2009;84:394.

17. Fortin C, Rouleau D. Cerebral Mycobacterium avium abscesses: Late immune reconstitution syndrome in an HIV-1-infected patient receiving highly active antiretroviral therapy. Can J Infect Dis Med Microbiol. 2005;16:187-9.

18. Calfee DP, Wespelwey B. Brain abscess, subdural empyema, and intracranial epidural abscess. Curr Infect Dis Rep. 1999;1:166-71.

19. Griffith DE, Aksamit T, Brown-Elliott BA, Catanzaro A, Daley C, Gordin F, et al. An Official ATS/IDSA Statement: Diagnosis, Treatment, and Prevention of Nontuberculous Mycobacterial Diseases. This official statement of the American Thoracic Society (ATS) and the Infectious Diseases Society of America (IDSA) was adopted by the ATS Board of Directors, September 2006, and by the IDSA Board of Directors, January 2007. Am J Respir Crit Care Med. 2007;175(4):367-416.
20. Nau R, Sorgel F, Eiffert H. Penetration of Drugs through the Blood-Cerebrospinal Fluid/Blood-Brain Barrier for Treatment of Central Nervous System Infections. Clin Microbiol Rev. 2010;23(4):858-83.

21. Mindermann T, Zimmerli W, Gratzl O. Rifampin Concentrations in Various Compartments of the Human Brain: A Novel Method for Determining Drug Levels in the Cerebral Extracellular Space. Antimicrob Agents Chemoter. 1998;42(10):2626-9.

\section{Submit your next manuscript to BioMed Central and take full advantage of:}

- Convenient online submission

- Thorough peer review

- No space constraints or color figure charges

- Immediate publication on acceptance

- Inclusion in PubMed, CAS, Scopus and Google Scholar

- Research which is freely available for redistribution

Submit your manuscript at www.biomedcentral.com/submit 